\title{
SCANNING BLAZED-GRATINGS FOR HIGH-RESOLUTION SPECTROSCOPY
}

\author{
Jocelyn T. Nee, Kam Y. Lau, and Richard S. Muller \\ Department of EECS \\ Berkeley Sensor \& Actuator Center \\ University of California at Berkeley \\ Berkeley, CA 94720-1774
}

\begin{abstract}
We have used anisotropic etching and subsequent polysilicon surface micromachining in a new process to produce actuated blaze gratings. Using the etch properties of $\mathrm{KOH}$ in single-crystal (100)-oriented silicon, we obtain repeatable $54.7^{\circ}$ blaze angles in the gratings by making use of the etched grooves as a mold for LPCVD-deposited polycyrstalline silicon. The designer can vary the grating period using conventional lithography techniques. After producing the grating structure in conventional surfacemicromachining steps, it is "folded" out from the surface and actuated by an on-chip electrostatic drive. We present experimental results made using prototype polysilicon gratings having an $11.7 \mu \mathrm{m}$ period in a square pattern $700 \mu \mathrm{m}$ on a side. We have also produced and assembled $1.3 \mathrm{~mm}$-tall grating structures that are now being tested. The polysilicon plates are mounted on torsion bars and actuated by electrostatic combdrives. If operated as high-order reflective gratings, these prototypes can be used to build integrated spectrometers with a resolving power of 1470 (wavelength resolution of approximately $0.4 \mathrm{~nm}$ ).
\end{abstract}

\section{INTRODUCTION}

Diffraction gratings are dispersive elements that are commonly used in optical instruments and systems. In addition, their ability to differentiate optical wavelengths makes diffraction gratings particularly suitable as switching components for wavelength-division multiplexing (WDM) communication systems or as wavelength selectors in tunable external-cavity laser systems.

We have previously demonstrated electrostatic comb-driven first-order rectangular gratings fabricated using MCNC's MultiUser MEMS Process (MUMPS) [1]. The resolution and diffraction efficiency of the rectangular gratings are limited by the achievable linewidth of the fabrication process. Because high resolving power and diffraction efficiency are required for WDM and tunable laser systems, the rectangular grating is not satisfactory. Blazed gratings, on the other hand, are not limited by the achievable linewidth and thus offer an alternative solution for improved resolution and diffraction efficiency. Blazed gratings are diffraction gratings with triangular diffracting surfaces (Figure 1), and they obey the grating equation,

$$
\mathrm{m} \lambda=\Lambda\left(\sin \theta_{\mathrm{m}}-\sin \theta_{\mathrm{i}}\right) .
$$

In Eq. (1), the diffraction angle $\theta_{m}$ and incident angle $\theta_{i}$ are measured from the grating normal (rather than the facet normal). Blazed gratings have large diffraction efficiency in the order of specular reflection, which is reflection of the light off the planar facet of each diffraction groove. In addition, since resolving power is proportional to the diffraction order, a larger resolving power is achievable due to the use of higher diffraction orders. Figure 2 shows SEM photograph of a combdrive-actuated blazed gratings that we have fabricated.

\section{DESIGN}

The electrostatic combdrive actuator for this system consists of 100 interdigitated fingers on the shuttle comb and 101 fingers on the stationary comb. The entire combdrive extended $1 \mathrm{~mm}$ in

\section{Diffracted beams}

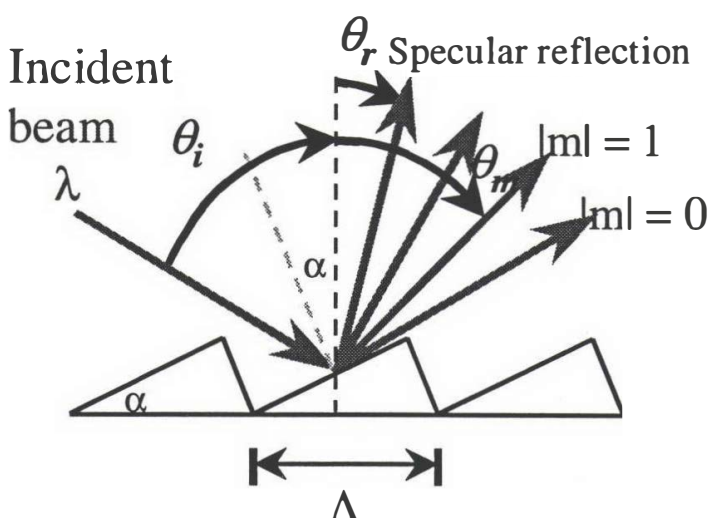

Figure 1. Schematic of a blazed diffraction grating and how it works. The period of the grating is $\Lambda$ and the blaze angle is $\alpha$.

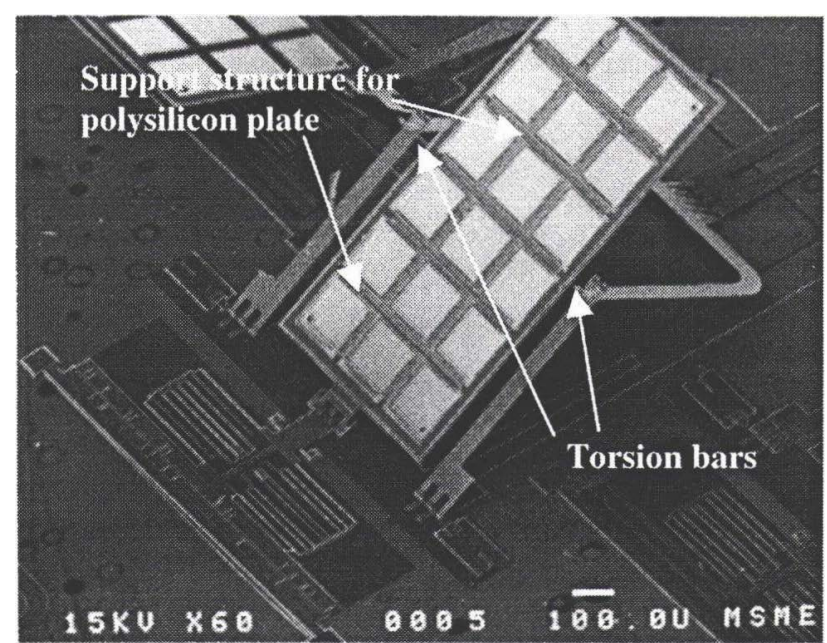

Figure 2. SEM of an actuated scanning blazed-grating. The diffracting surface is on the back side of the plate. The grid structure on the front side is a support structure for the thin polysilicon plate.

total width, with comb fingers $2 \mu \mathrm{m}$ wide, $2 \mu \mathrm{m}$ thick, and $40 \mu \mathrm{m}$ long. The combdrive is hinged to the bottom of the grating by a cross-woven polysilicon-stripe assemblage to ensure firm locking of the joint. The gratings are mounted on the frames by two 50 $\mu \mathrm{m}$-long torsion bars (with cross-sectional areas of $2 \times 2 \mu \mathrm{m}^{2}$ ) as the rotational shafts. The dimensions of the polysilicon plates are $700 \mu \mathrm{m} \times 700 \mu \mathrm{m}$ for the small plates and $700 \mu \mathrm{m} \times 1300 \mu \mathrm{m}$ for the large plates. The actual areas of the grating on the plates are $550 \mu \mathrm{m} \times 500 \mu \mathrm{m}$ and $550 \mu \mathrm{m} \times 1050 \mu \mathrm{m}$ for small and large plates respectively. 


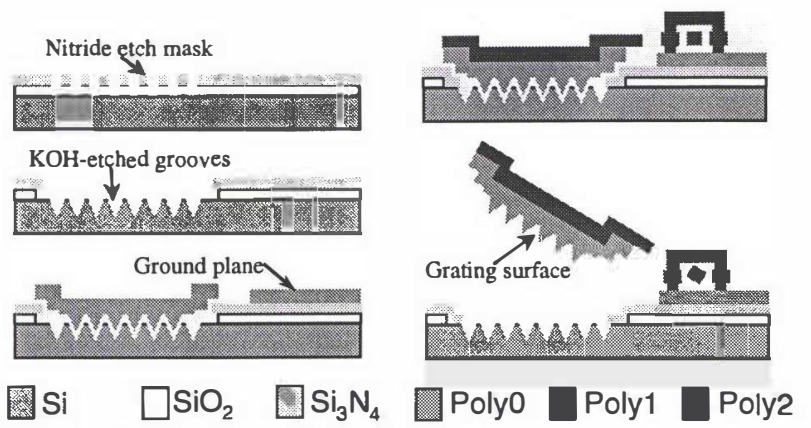

Figure 3. Cross section of the processing sequence for fabricating blazed diffraction gratings using anisotropically etched silicon substrate as mold. The grated polysilicon plate is defined in the PolyO layer, and the support structure (crisscrossed beams) is defined in the Polyl layer.

\section{FABRICATION}

The blazed diffraction gratings are fabricated in the UCB Microfabrication Laboratory using a new fabrication process, which combines a pre-process of anisotropic $\mathrm{KOH}$ etching of silicon and the traditional two-structural polysilicon surfacemicromachining process. The processing sequence for the blazed gratings is shown in Figure 3. The mold for the grating surface is formed by parallel V-grooves etched in (100) silicon substrate using anisotropic $\mathrm{KOH}$ etching. A total of $499.7-\mu \mathrm{m}$-wide grooves, spaced by $2 \mu \mathrm{m}$, are etched in the silicon substrate. The grating has a blaze angle $\alpha$ of $54.7^{\circ}$ as defined by anisotropic $\mathrm{KOH}$ etching of (100) silicon. A sacrificial LPCVD silicon dioxide (PSG) layer is deposited on top of the blazed grating mold. The $0.5-\mu \mathrm{m}$ polysilicon layer (Poly0) deposited in the next step is then "molded" by these grooves and thus forms the grating surface. Support for the thin grating plate is provided by criss-crossed support beams in the first $2-\mu \mathrm{m}$ structural polysilicon layer (Poly1). SEM photographs of the edge and surface of the grating surface are shown in Figure 4. The electrostatic combdrives are defined in the Polyl layer, as are the frame and the torsion bars connecting the grating plate to the frame.

\section{CHARACTERIZATION \& DISCUSSION}

We have measured the diffraction pattern of the blazed grating, as shown in Figure 5. Only the diffraction orders adjacent to the specular order are significant. We have also demonstrated non-resonance scanning of the grating. The mechanical design allows the shuttle comb to travel a distance of $5 \mu \mathrm{m}$ in the nonresonant mode, which translates into a scanning angle of approximately $10^{\circ}$ for a grating that is hinged at $50 \mu \mathrm{m}$ above the substrate. Detailed characterization of the optical properties will be reported.

\section{CONCLUSION}

We have constructed novel scanning blazed gratings for highresolution spectroscopy. Out-of-plane blazed gratings with dimensions as large as $700 \mu \mathrm{m} \times 1300 \mu \mathrm{m}$ were fabricated using a new surface-micromachining process which we have developed. These remarkably large, thin plates are actuated by electrostatic combdrives and are structurally supported by wide polysilicon beams. Use of these blazed gratings, which have a wavelength resolution of roughly $0.4 \mathrm{~nm}$ and a scan angle of $10^{\circ}$, in a scanning spectrometer will enable resolution of approximately 400 distinct wavelengths.
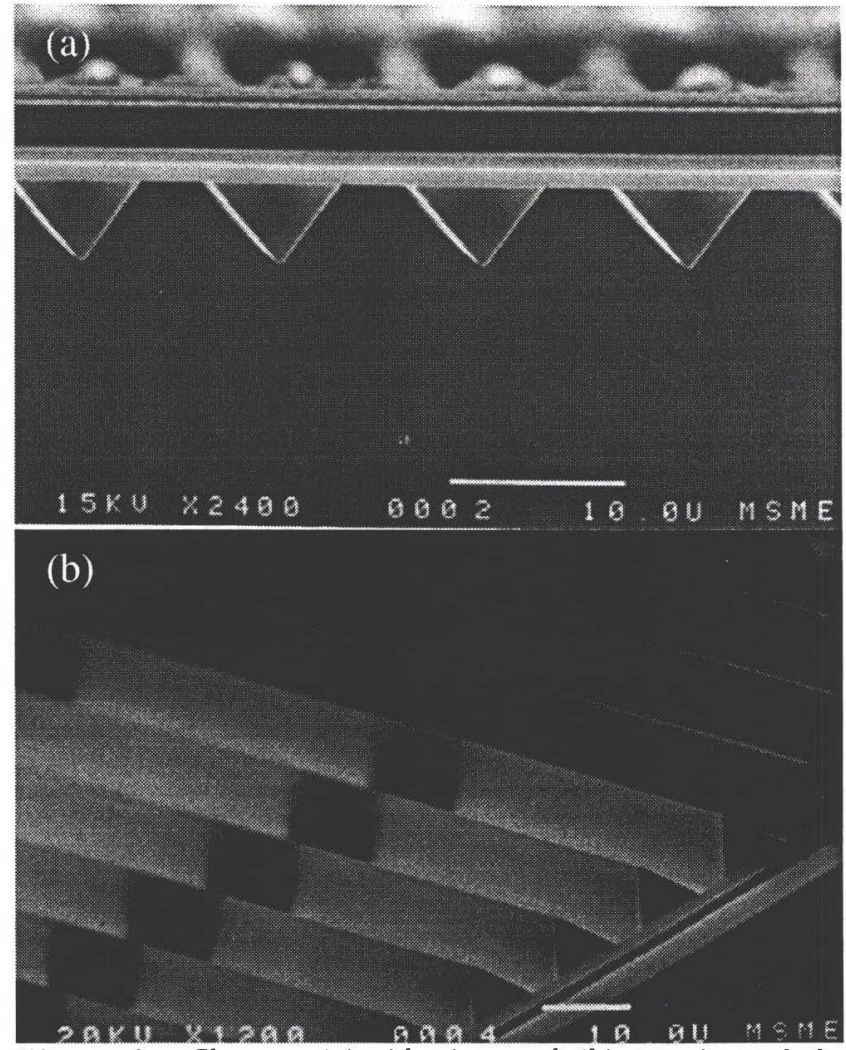

Figure 4. Close-up (a) side-view and (b) top-view of the diffracting surface of the blazed grating. The cross-section is triangular in shape.

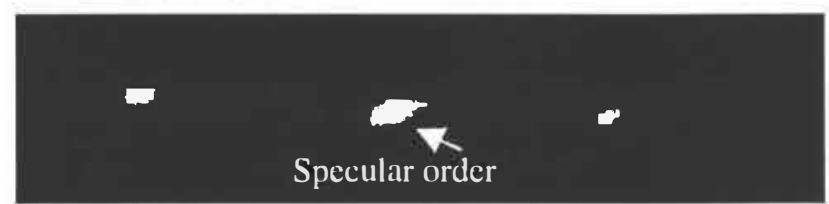

Figure 5. Diffraction pattern of the blazed grating. Diffraction orders not adjacent to the specular order are very small compared to the specular order.

\section{ACKNOWLEDGEMENTS}

The authors thank Ron Wilson and Wen-Chin Lee for help in obtaining SEM pictures, and Janice Hudgings for assistance with optical measurements.

This material is based upon work supported under a National Science Foundation Graduate Fellowship and DARPA grant contract number DABT-63-95-C-0055.

\section{REFERENCES}

1. M.-H. Kiang, J. T. Nee, K. Y. .Lau, and R. S. Muller, "Surface-Micromachined Diffraction Gratings For Scanning Spectroscopic Applications", 1997 International Conference on Solid State Sensors and Actuators (Transducers'97) Digest of Technical Papers, Chicago, IL, USA,6/16-19/97, Transducer Research Foundation, Cleveland (1997), vol. 1, pp. 343-345. 